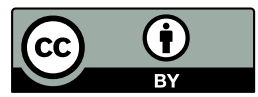

\title{
Conocimiento ecológico tradicional de la biodiversidad de bosques en una comunidad matlatzinca, México*
}

\section{Traditional Ecological Knowledge of Forests Biodiversity in a Matlatzinca Community, Mexico}

\section{Connaissances écologiques traditionnelles de la biodiversité des forêts d'une communauté Matlatzinca, Mexique}

\section{Laura Millán-Rojas**, Tizbe T. Arteaga-Reyes***, Sergio Moctezuma-Pérez****, Juan Jesús Velasco-Orozco*****, José Concepción Arzate-Salvador ${ }^{* * * * * *}$}

Recibido: 2015-07-10 // Aprobado: 2015-08-12 // Disponible en linea: 2016-01-30

Cómo citar este artículo: Millán-Rojas, L., Arteaga-Reyes, T. T., Moctezuma-Pérez, S., Velasco-Orozco, J. J. y Arzate-Salvador, J. C. (2016). Conocimiento ecológico tradicional de la biodiversidad de bosques en una comunidad matlatzinca, México. Ambiente y Desarrollo, 20(38), 111-123. http://dx.doi. org/10.11144/Javeriana.ayd20-38.cetb doi:10.11144/Javeriana.ayd20-38.cetb

\section{Resumen}

La inclusión del conocimiento ecológico tradicional sobre la biodiversidad en la toma de decisiones, es prioritaria para garantizar el respeto de los conocimientos tradicionales y conjuntarlo con los conocimientos científicos para la protección y conservación de los recursos naturales. El objetivo del presente estudio fue documentar el conocimiento ecológico tradicional que posee un grupo indígena del altiplano central de México. Se emplearon metodologías cualitativas, destacando observación directa, entrevistas y un taller participativo. Los resultados obtenidos demuestran que hay una continuidad del conocimiento ecológico tradicional basada en aspectos culturales. Este conocimiento junto con las creencias y prácticas de los habitantes

* Artículo de investigación derivado del proyecto de investigación Conocer para conservar: la biodiversidad, un servicio ambiental en el Nevado de Toluca, clave 3561/2013cHT, financiado por la Universidad Autónoma del Estado de México a través de la Secretaría de Investigación y Estudios Avanzados.

** Licenciada, Universidad Autónoma del Estado de México, Instituto de Ciencias Agropecuarias y Rurales, El Cerrillo Piedras Blancas, 50090, Toluca, Estado de México, México. Estudiante de posgrado. Correo electrónico: lauramillann@gmail.com

*** Doctora, Universidad Autónoma del Estado de México, Instituto de Ciencias Agropecuarias y Rurales, El Cerrillo Piedras Blancas, 50090, Toluca, Estado de México, México. Profesora-investigadora. Autora para la correspondencia. Correo electrónico: tizbe@hotmail.com

**** Doctor, Universidad Autónoma del Estado de México, Instituto de Ciencias Agropecuarias y Rurales, El Cerrillo Piedras Blancas, 50090, Toluca, Estado de México, México. Profesor-investigador. Correo electrónico: sergiomoctezuma@hotmail.com

***** Doctor. Universidad Autónoma del Estado de México, Facultad de Antropología, Mariano Matamoros s/n, Col. Universidad, 50130, Toluca, Estado de México, México. Profesor-investigador. Correo electrónico: jujevo@gmail.com

****** Maestro, Universidad Autónoma del Estado de México, Facultad de Antropología, Mariano Matamoros s/n, Col. Universidad, 50130, Toluca, Estado de México, México. Profesor-investigador. jc_ar75@hotmail.com 
influye en los valores doméstico, económico, medicinal, ritual y ontológico otorgados a la flora y fauna de sus bosques.

Palabras clave: conocimiento ecológico tradicional; cambio cultural; biodiversidad; indígena; área natural protegida

\begin{abstract}
The inclusion of the traditional ecological knowledge regarding the biodiversity, into the decision-making is fundamental in order to guarantee the respect of traditional knowledge and to link it to scientific knowledge for protection and conservation of natural resources. The objective of the current study was to document traditional ecological knowledge of an indigenous group in the Central Highlands of Mexico. Qualitative methodologies were employed highlighting direct observation, interviews and a participatory workshop. Results show that there is a continuity of traditional ecological knowledge based on cultural aspects. This knowledge, together with believes and practices of inhabitants, influences on domestic, economic, medicinal, ritual and ontological values attributed to flora and fauna of their forests.
\end{abstract}

Keywords: traditional ecological knowledge; cultural change; biodiversity; indigenous; natural protected area

\title{
Résumé
}

La prise en compte des connaissances écologiques traditionnelles sur la biodiversité est essentielle dans les prises de décision, pour garantir le respect de ces connaissances et afin de les croiser avec les connaissances scientifiques portant sur la protection et la conservation des ressources naturelles. L'objectif de ce travail est d'étudier les connaissances écologiques traditionnelles d'un groupe indigène du haut-plateau central mexicain. Des méthodologies qualitatives ont été employées à travers des observations directes, des entretiens et un atelier participatif. Les résultats obtenus montrent qu'il existe une continuité culturelle des connaissances écologiques traditionnelles. Ces connaissances ainsi que les croyances et pratiques des habitants influent sur les valeurs domestiques, économiques, médicinales, rituelles et ontologiques conférées à la faune et à la flore des forêts.

Mots clés: connaissance écologique traditionnelle ; changement culturel ; biodiversité; indien ; aire naturelle protégée 


\section{Introducción}

En la actualidad, las sociedades indígenas de México se encuentran en un proceso de cambio cultural, originado por el impacto de la modernización y la adopción de estilos de vida característicos de las actuales sociedades urbanas. Lo anterior se expresa de manera particular a través de la pérdida, el desuso y la modificación de prácticas orientadas a la subsistencia, que en otras épocas implicaban el uso de especies vegetales. Ante esta situación, resulta pertinente describir la dinámica del cambio cultural, a partir de las continuidades y los cambios en el uso y conocimiento de la flora y fauna local. Con base en lo anterior, el concepto de conocimiento ecológico tradicional (TEK, por sus siglas en inglés) permite analizar los conocimientos, las prácticas y las creencias que poseen los grupos humanos acerca de la relación entre los seres vivos de un determinado ambiente, así como su transmisión hacia las nuevas generaciones (Berkes, 1999).

Los estudiosos de las culturas tradicionales contemporáneas tienen la importante tarea de documentar, analizar y revalorar los conocimientos premodernos que poseen las culturas sobre la naturaleza (Toledo, 2005); es necesario un diálogo entre diversas ciencias occidentales — como la antropología ecológica y las ciencias agropecuarias - que parta del principio de respeto hacia los conocimientos empíricos que aún perviven, porque estos forman parte de las estrategias de subsistencia de una gran cantidad de culturas.

Este diálogo posibilita el cumplimiento de los principios de integración y reconocimiento del TEK que también promulgan instancias internacionales de la conservación. Tal es el caso de la Organización de las Naciones Unidas para la Educación, la Ciencia y la Cultura (Unesco) y la Comisión de las Naciones Unidas para el Medio Ambiente que desde la década de 1990 lo reconocen en el Convenio de Diversidad Biológica, el cual establece que el conocimiento que poseen los grupos indígenas debe ser respetado, preservado y aplicado, puesto que permite la conservación y la utilización sostenible de la diversidad biológica (Naciones Unidas, 1992). La Unión Internacional para la Conservación de la Naturaleza (IUCN) considera que el TeK es una forma eficaz de adaptación ante el cambio climático y al mismo tiempo puede ser la base para el diseño de políticas públicas de escala nacional e internacional (Lara y Vides, 2014).

Por tanto, se enfatiza que la documentación del TEK tiene un valor científico per se y permite demostrar que es posible llevar a cabo un uso y manejo de los recursos naturales de manera sostenible; su documentación en grupos indígenas y la identificación de la capacidad de resiliencia de los sistemas socioecológicos donde habitan, son dos pasos necesarios para que la ciencia occidental pueda ser escuchada por los hacedores de políticas públicas a diferentes escalas. Se basa en la premisa de que la conservación de la biodiversidad y el conocimiento aportan beneficios a la comunidad que los posee, y que se extienden más allá de las simples categorías territoriales, que pueden resultar estáticas si no se les conceptualiza como parte de unidades geográficas mayores.

El presente trabajo se centra en documentar el TEK que posee un grupo indígena matlatzinca del altiplano central de México en San Francisco Oxtotilpan, estado de México, sobre la biodiversidad existente en sus bosques dentro del Área de Protección de Flora y Fauna Nevado de Toluca. Las dos características anteriores posibilitan que este grupo indígena sea un referente importante en cuanto a conocimiento ecológico tradicional.

\section{Consideraciones generales en torno al TEK}

El TEK de los grupos indígenas es principalmente cualitativo, intuitivo, holístico, moral y espiritual, basado en la observación empírica y la acumulación de hechos a través del uso cotidiano de los recursos naturales (Berkes, 1993). Esto implica una estrecha relación entre la sociedad y su ambiente y puede ser el cimiento para un uso sustentable de dichos recursos basado en una cosmovisión compartida. La descripción del conocimiento tradicional — prácticas, valores, creencias, cosmovisión- que poseen las sociedades indígenas siempre ha sido un campo de interés para la antropología social. Desde la década de 1970, dichas 
descripciones se focalizaron principalmente en el TEK, dando origen a una antropología cognitiva que a su vez se dividió en etnociencias: etnoecología, etnobiología, etnomedicina, entre otras (Milton, 1996).

Los estudios que se realizan en comunidades indígenas deben enfocarse en describir las tres variables que conforman el TeK: 1) conocimientos, 2) prácticas y 3) creencias. De acuerdo con Menzies y Butler (2006), el conocimiento es acumulativo y es el resultado de un proceso histórico; por tanto, se deben estudiar las formas de transmisión del conocimiento entre las generaciones. Generalmente, es a través de la transmisión oral y de manera particular mediante la narración de cuentos, mitos y leyendas, metáforas o explicaciones sobre cómo realizar alguna actividad de la vida cotidiana.

Asimismo, el conocimiento se puede transmitir cuando los adultos o poseedores de este solicitan a los jóvenes la realización de una actividad específica o instruyen sobre cómo realizarla (Corsiglia, 2006). Es necesario que el conocimiento que documenta el investigador se exprese en las categorías locales con que cuentan los grupos étnicos, enfocándose en entender las formas para nombrar los componentes del ambiente natural y cultural (Townsend, 2009).

Las prácticas están asociadas a procesos habituales, es decir, que se realizan de manera continua desde tiempo atrás. En ocasiones, no es posible ubicar temporalmente el inicio de una práctica, pero sí se puede indagar por los cambios, continuidades e innovaciones que provienen de diferentes escalas — local, regional, estatal, nacional o internacional - y que permiten la adaptabilidad de la sociedad a diferentes contextos — sociales, políticos, económicos, culturales - en los cuales las prácticas pueden modificarse para su exitosa adaptación o desaparición (Menzies y Butler, 2006).

Las prácticas se materializan en actividades vinculadas estrechamente con la consecución del sustento (Menzies, 2006), es decir, en prácticas agrícolas. Por ejemplo, los rituales para propiciar la fertilidad de la tierra, y por lo tanto, el incremento en la producción de alimentos (Mariaca et al., 2014). De esta forma, las prácticas justifican la importancia de conocer las interacciones que existen en el ambiente y de aplicarlas ampliamente a la obtención de dicho sustento.

Las creencias forman parte de la cosmovisión de los grupos indígenas; es la variable del TEK considerada como la amalgama entre el conocimiento y la práctica: es la razón de conocer y hacer, es decir, son el fundamento epistémico de una cultura (Toledo y Barrera, 2009). Para Menzies y Butler (2006) las creencias tienen una fundamentación moral y espiritual. Lo anterior indica a los individuos lo que pueden y no pueden hacer dentro de los ecosistemas que habitan, e incluso hasta las formas de alimentarse, dependiendo de su ciclo de vida (Magallanes et al., 2005).

En México, el estudio y la comprensión del TEK es imprescindible para discutir el potencial ecológico, social, cultural y económico de la flora y la fauna que se encuentran en los territorios habitados por grupos indígenas. Por ejemplo, LaRochelle y Berkes (2003) describen cómo los indígenas rarámuris del norte de México se consideran a ellos mismos emparentados con la naturaleza. Esta relación se refleja en la práctica de recolectar solamente las plantas comestibles necesarias; existe una lógica sustentable para no agotar alguna especie vegetal comestible y para monitorear su disponibilidad, resultado de la observación y el entendimiento de procesos ecológicos. Implica que cuando estos conocimientos alcanzan una fundamentación empírica, deben ser transmitidos a las nuevas generaciones a través del lenguaje. Para los rarámuris, aprender la lengua y entender el ambiente físico, es parte de un mismo sistema de enseñanza local.

La cosmovisión de los grupos indígenas puede representarse en el número de plantas que la población reconoce, recolecta, almacena y consume. Por ejemplo, Quiñonez et al. (2014) reportan que los rarámuris del estado de Chihuahua reconocen y consumen 16 especies de hongos presentes en su entorno. Este conocimiento es transmitido principalmente por los padres y abuelos a las generaciones más jóvenes. Para el caso de los mayas de la península de Yucatán, Santos et al. (2012) documentan el uso y conocimiento de 46 especies de animales con fines alimenticios, medicinales, comerciales, ornamentales y para la elaboración de herramientas, entre otros. La cacería de estos animales involucra el conocimiento sobre determinadas prácticas y técnicas. Por lo anterior, el TEK puede considerarse 
un sistema cultural basado en un proceso de enseñanza y aprendizaje a través de símbolos y de la acumulación de información (Manfredo et al., 2014).

El TEK se relaciona con la capacidad de adaptabilidad del ser humano a su ambiente, quien puede ajustar su cultura y sus comportamientos ante eventuales cambios (Moran, 2000); existe y pervive solamente a partir de su transmisión, la cual depende a su vez de la utilidad que tenga para la población que lo posee (Berger y Luckmann, 2011). Cuando los individuos encuentran una nueva manera de realizar una actividad, se apropian de ella, siempre y cuando implique un ahorro de energía o de tiempo, incluso sin importar que la actividad que se reemplaza esté cargada de una connotación cultural o identitaria. Por ejemplo, la escolaridad y el trabajo asalariado son dos factores que provocan el deterioro del теK entre los zapotecos de Oaxaca, toda vez que genera expectativas y necesidades que forman necesidades asociadas a una vida de tipo urbano, moderno e industrializado y que solo pueden satisfacerse mediante una completa incorporación al sistema de mercado. De esta forma, por ejemplo, el uso de plantas medicinales en comparación con el uso de medicamentos, en una asociación dicotómica de tradicional/moderno (Saynes et al., 2013).

El rescate y conservación del TEK permite mantener la diversidad biológica y cultural contenida en un ecosistema. Al mismo tiempo, es una alternativa ante productos y procesos que forman parte de una economía de mercado, a la cual, por condiciones históricas, las comunidades indígenas se integran en desventaja. La documentación del TEK y su promoción pueden ayudar a generar programas de desarrollo que tomen en cuenta los recursos locales y promuevan el desarrollo bajo un modelo basado en la conservación (Ruiz y Corbera, 2013).

\section{San Francisco Oxtotilpan: residencia del grupo indígena matlatzinca en el altiplano central de México}

En el altiplano central de México habita en San Francisco Oxtotilpan, municipio de Temascaltepec, estado de México (figura 1), la única y última comunidad indígena matlatzinca, de aproximadamente 1435 habitantes en el 2010 (Borboa, 1999; Instituto Nacional de Estadística y Geografía (Inegi), 2010; Quezada, 1996), de los cuales 731 aún hablan la lengua matlatzinca (Gaceta Municipal [GEM], 2013). Parte de su territorio se ubica en el Área de Protección de Flora y Fauna Nevado de Toluca (APFF NT) y corresponde a la Región Prioritaria para la Conservación 109 Nevado de Toluca, caracterizada por ecosistemas de alta montaña con bosques de pino (Pinus spp.), oyamel (Abies religiosa) y encino (Quercus spp.), de los cuales se obtienen diversos recursos naturales (Comisión Nacional de Áreas Naturales Protegidas (Conanp), 2014).

\section{Materiales y métodos}

Se utilizó metodología cualitativa, considerando el carácter comunitario de San Francisco Oxtotilpan, dado que el objetivo de la investigación fue documentar el тек. Esta metodología permitió un acercamiento a profundidad a través de la observación directa, entrevistas (dirigidas y semiestructuradas) y un taller participativo. La observación directa tuvo lugar en recorridos en los bosques de San Francisco Oxtotilpan en el mes de abril de 2014, durante acompañamientos en actividades relacionadas con biodiversidad (faenas y colecta ritual). En estos recorridos participaron habitantes registrados en el padrón de ejidatarios activos y se realizaron con la finalidad de identificar y documentar las diversas especies de flora y fauna que utilizan y se encuentran en sus bosques, a través de registros visuales como fotografías para el caso de flora y menciones por parte de los habitantes de los nombres de los animales que ellos han observado en los bosques de su comunidad. Las entrevistas dirigidas se realizaron a los participantes durante los recorridos en campo y a informantes clave nominados mediante el muestreo no probabilístico "bola de nieve"; estas incluyeron rubros respecto a creencias, conocimientos y prácticas relacionadas con la biodiversidad de sus bosques. 


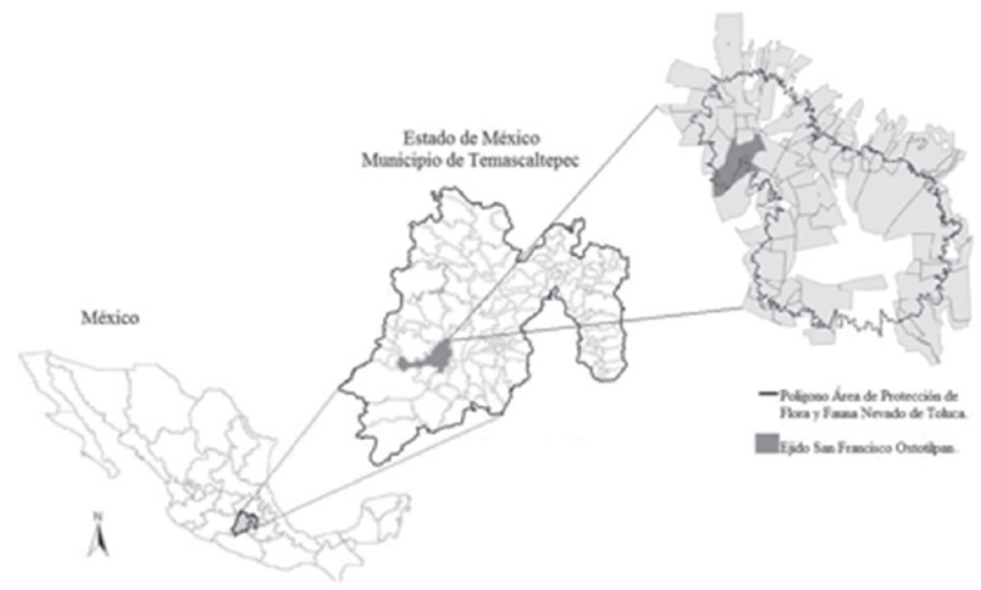

Figura 1. Ubicación de San Francisco Oxtotilpan

Fuente: modificado por Laura Millán (2015)

En el mes de mayo del 2014 se realizó un taller participativo con mujeres pertenecientes a la comunidad, con el objetivo de identificar los valores atribuidos a las especies de flora; la invitación a participar en dicho taller fue abierta tanto a hombres como a mujeres. La dinámica consistió en mostrarles en una computadora imágenes (fotografías) digitales a color de las especies de flora, previamente fotografiadas e identificadas en los recorridos de campo por otros informantes clave. Se realizó una entrevista grupal dirigida, considerando los siguientes rubros: identificación solo con imagen, identificación con nombre, usos conocidos, formas de utilización y valores o importancia atribuida.

Las entrevistas semiestructuradas se aplicaron en el mes de junio del 2014, de manera aleatoria a habitantes de la misma comunidad, con el fin de corroborar la información obtenida durante los recorridos en los bosques. Se enfatizó en: la fauna existente, es decir, la conocida o vista en sus bosques; la disminución o pérdida de especies; los usos, propiedades o significados atribuidos a dicha fauna, así como su importancia.

\section{Resultados y discusión}

El análisis del TEK de la comunidad matlatzinca permite observar que sus habitantes cuentan con un conocimiento compartido de la naturaleza, que se ha transmitido y conservado por generaciones y se refleja en el reconocimiento de las especies, en la tradición oral respecto a sus usos (sean o no vigentes) y en el valor de uso otorgado: doméstico, religioso o ritual, medicinal y económico.

La transmisión del conocimiento respecto a la biodiversidad de los bosques está acompañada de la sucesión de responsabilidades, como en el caso de los derechos ejidales de padres a hijos o hijas, o de la sucesión de cargos cívicos o religiosos entre adultos de la comunidad. El relevo de derechos ejidales ocurre con el fallecimiento del titular, pero el proceso de transmisión sucede bajo un entendimiento de que este se generará durante la edad adulta del heredero o heredera; por tanto, la adultez es una de las condiciones para ser acreedor de cargos cívicos o religiosos cuando se es padre de familia.

Es importante recalcar que también las enseñanzas sobre las características, usos y propiedades de algunas especies de flora y fauna, que se suscitan dentro de la vida cotidiana, forman parte del proceso de transmisión. Por ejemplo, el uso de algunas plantas como el tomatillo para la preparación de alimentos, así como los procesos de recolección y preparación de los hongos con el mismo fin, son prácticas de la cotidianidad que son conocidas y replicadas por las nuevas generaciones. 
Las prácticas como medio de aplicación del conocimiento sobre la biodiversidad giran en torno a los bosques; por ejemplo, las faenas, ${ }^{1}$ recorridos de vigilancia del bosque, recolección de recursos maderables y no maderables y prácticas de recreación. Las creencias pueden estar basadas en experiencias empíricas de quienes las transmiten y en el reconocimiento de dichas experiencias como verdad por parte de quienes se las apropian. Por ejemplo, la creencia heredada de madres a hijas de los efectos medicinales que tienen algunas plantas durante el proceso de lactancia, que llevan a las mujeres que no producen suficiente leche materna a colocarse en la espalda una planta conocida como jarilla (previamente "sudada", que es el proceso de ponerla sobre un comal al fuego) con el fin de generar mayor cantidad de leche.

Es así como el TEK genera, además, un proceso de valoración que está ligado a la obtención del sustento de la vida cotidiana como la alimentación, a la continuación de una creencia que puede ser religiosa o no, y a la explicación de una realidad que da lugar al uso de ciertas especies para fines específicos.

En este contexto, se resumen y explican los valores que la población de San Francisco Oxtotilpan otorga a la biodiversidad de sus bosques. El valor doméstico es otorgado por los atributos que permiten el uso de diversas especies de plantas y recursos forestales maderables (ocote y pino) en las actividades asociadas a las labores domésticas como la preparación de alimentos —que requiere combustible—, leña, o como materia prima para la construcción de muebles. El valor medicinal se refiere a la importancia que representan las plantas para los habitantes por los atributos reconocidos y experimentados en ellas para el alivio de diversos padecimientos fisiológicos como gripe, diabetes, dolor de cabeza y aquellos asociados a los riñones.

El valor religioso o ritual se refiere a la importancia adquirida por la transmisión de conocimientos y experiencias en cuanto a los procesos rituales que se llevan a cabo; representa una importancia espiritual y de respeto por parte de los habitantes por su uso en actividades religiosas como fiestas patronales, petición de un buen temporal para el ciclo agrícola, Día de Muertos y Semana Santa.

El valor económico es atribuido por el beneficio económico que representa para sus habitantes a través de la comercialización, por ejemplo, de plantas como el carricillo y algunas especies de hongos a muy baja escala, así como de recursos maderables mediante un aprovechamiento forestal, que representa una fuente de ingreso (tabla 1).

Algunas prácticas han desaparecido debido a prohibiciones o a la dificultad para tener acceso a los recursos naturales. Se incentivó o sensibilizó a la comunidad sobre erradicar el consumo de hongos alucinógenos como los "santitos" (chotamí o tochutata en matlatzinca). La población atribuye a los "santitos" propiedades curativas y mágicas o de predicción; se cree que si este hongo era consumido por una persona enferma, ante ella aparecía "alguien" que le consultaba sobre sus síntomas y le informaba si su enfermedad tenía o no cura; también consideran que en el caso de que alguien haya perdido un animal — de corral — en el bosque, el consumo del hongo les permite adivinar dónde se encuentra.

Existen plantas como la salvia que es recolectada por las mujeres, quienes la usan en los temascales con el fin de generar calor en el cuerpo, así como para limpiar o bañar a las mujeres recién "aliviadas", o bien como infusión para curar enfermedades respiratorias — como la gripe-; algunas reconocen que aprendieron a preparar y aplicar la salvia mediante un baño de hierbas.

Respecto a las especies faunísticas, los testimonios (obtenidos en los recorridos, entrevistas y en el taller participativo), experiencias y conocimientos de los pobladores de esta comunidad permitieron identificar únicamente un valor cultural y realizar un registro de la fauna existente en sus bosques (tabla 2), pero no fue posible obtener un acervo fotográfico durante los recorridos en los bosques, porque los animales tienden a alejarse de la gente, además de que para algunos su actividad es generalmente nocturna. La fauna representa para los habitantes un valor ontológico, es decir, es importante: 1) porque forma parte de un ecosistema o "son parte de la naturaleza"; los animales que habitan en el bosque tienen una razón de ser y de existir; y 2) porque influye en el estado de ánimo y

1 Trabajo colectivo que busca un bien común; es obligado y no remunerable. 
Tabla 1. Tipo de valores para flora

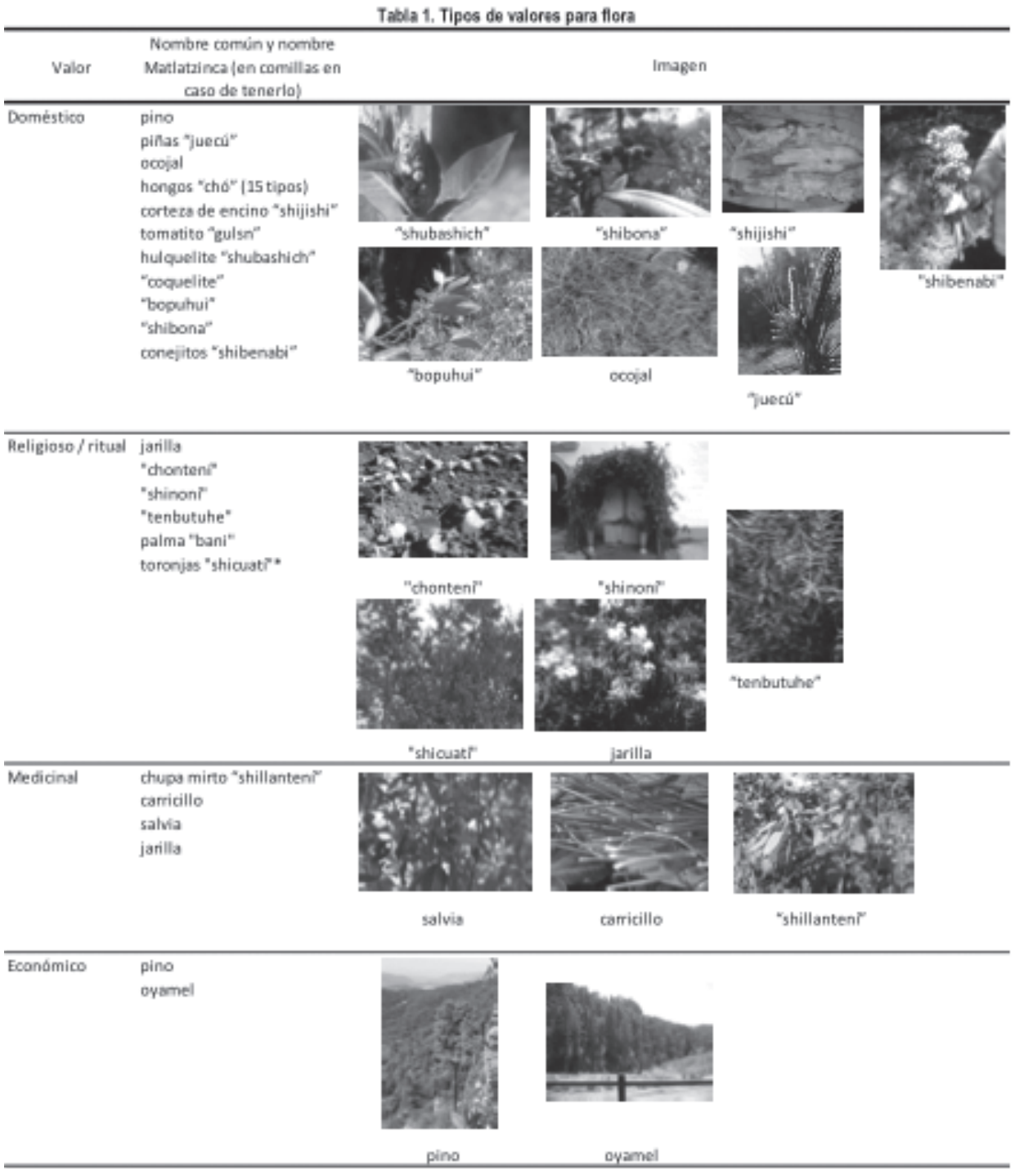

Nota: el registro escrito de los nombres de la flora en lengua matlatzinca se basó en la comprensión auditiva del entrevistador, con ayuda de los mismos hablantes de San Francisco Oxtotilpan.

* Nombre que los habitantes de San Francisco Oxtotilpan utilizan para referirse a las agallas que se forman cuando una avispa hembra, de la familia Cynipidae, deposita sus huevos en una hoja o en cualquier otra estructura del encino. Las agallas sirven para proteger y facilitar el desarrollo de las larvas.

Fuente: elaboración propia 
Tabla 2. Conocimiento ecológico tradicional de la fauna en San Francisco Oxtotilpan

\begin{tabular}{|c|c|}
\hline Nombre común & Nombre en matlatzinca \\
\hline Venado & Mapinu \\
\hline Conejo & Ncuá \\
\hline Ardilla & Miní \\
\hline Víbora (cascabel, correlona, dormilona) & Chiní \\
\hline Ratón & Shupó \\
\hline Armadillo & Jtobi \\
\hline Zorrillo & Nilbi \\
\hline Tlacuache & Simuachir \\
\hline Tejón & Shuyoní \\
\hline Búho & Cujcú \\
\hline Correcaminos & Nilce \\
\hline Lagartija & Yiconí \\
\hline Mariposa & Shimuwi \\
\hline Alicante & --------- \\
\hline Escorpión, alacrán & ---------- \\
\hline Abeja & Spá \\
\hline Salamandra & Menoní \\
\hline Coyote & Suyowi \\
\hline $\begin{array}{c}\text { Pájaro (carpintero, jilguero, mulato, pojcohui, } \\
\text { nsayogui) }\end{array}$ & Rutaní \\
\hline Camaleón & -...-.-.-.- \\
\hline ------------------ & $\mathrm{Jaz}^{*}$ \\
\hline
\end{tabular}

Nota: el registro escrito de los nombres de la fauna en lengua matlatzinca se basó en la comprensión auditiva del entrevistador, con ayuda de los mismos hablantes de San Francisco Oxtotilpan.

* Este animal recibe su nombre por el sonido que emite. Pocas personas lo han visto y lo describen como un animal parecido a un perro.

Fuente: elaboración propia

permite una gratificación de los sentidos; son depositarios de alegría y belleza como en el caso de las mariposas, el conejo, el venado y las aves —nsayoni ${ }^{2}$ y pojcohui (2001). Entre las especies de fauna identificadas por los habitantes se encuentran aquellas a las que se les atribuye o atribuían propiedades para su uso y aprovechamiento y aquellas que son portadoras de significados culturales como el zorrillo, ${ }^{4}$ el tlacuache, el coyote, la víbora de cascabel y el búho.

2 Esta ave es considerada de mal augurio; su avistamiento representa una señal de que algo malo le va a ocurrir a la persona que la vio.

3 Se cree que escuchar el sonido de esta ave es señal de que esa persona recibirá una visita.

4 Para este animal solamente se documentó que su carne era apreciada por su valor para curar el mal de 
Es importante mencionar que la vigencia de los usos de flora y fauna en San Francisco Oxtotilpan se ha visto influida en gran medida por un largo proceso de "modernización" (Giménez, 1995). La trasmisión cultural del TEK en esta comunidad indígena ha evitado su desaparición; sin embargo, dicho conocimiento se puede ver amenazado por la falta de aplicabilidad y disminución de contacto entre los habitantes de las nuevas generaciones con su medio natural, resultando en la indiferencia respecto a la disminución de especies o la pérdida del mismo conocimiento. Por ejemplo, los usos y propiedades que se les atribuían a ciertas especies como el huiquilite, una planta anteriormente utilizada para lavar la ropa por sus propiedades blanqueadoras, no han sido olvidados; sin embargo, ya no es utilizada, debido al acceso a productos comerciales que facilitan el fin que cumplía esta hace más de treinta años. Lo mismo ocurre con las plantas a las que se les atribuyen propiedades medicinales; en la actualidad este conocimiento persiste y contribuye al rescate del TEK respecto a las propiedades de diversas especies. A pesar de que las nuevas relaciones de mercado hayan modificado la estructura tradicional, esta comunidad indígena conserva una relación importante con su biodiversidad (Latapí, 2009); existe una continuación y transmisión del TEK a las nuevas generaciones, por ejemplo, gracias a su inclusión en las prácticas relacionadas con los bosques, los jóvenes adquieren conocimientos derivados de hechos circunstanciales que posiblemente apliquen en su edad adulta.

En la comunidad de San Francisco Oxtotilpan la lengua es un referente de la supervivencia del TeK y de la continuación de los usos que le dan a la biodiversidad de sus bosques; se identifican los valores y significados otorgados a la biodiversidad, valores que no se pueden cuantificar pero que existen, se transmiten y refuerzan la relación hombre-naturaleza. El lenguaje es el acto de conocer (Hornborg, 2001); es decir, que la continuación y reconstrucción de una cultura encuentra en la lengua un vehículo de supervivencia.

La organización e interacción social juegan un papel importante en la supervivencia de los conocimientos y las prácticas, debido a que permiten la toma de decisiones y acciones en cuanto al manejo de los recursos naturales. Los matlatzincas realizan acuerdos y llevan a cabo actividades dentro de los bosques tales como faenas para la construcción de refugios de especies, brechas cortafuegos, rondines de vigilancia, limpieza y extracción de recursos maderables, colectas rituales, entre otras. Estas actividades se convierten en un medio de integración que posibilita la generación y la transmisión del TEK en la praxis, y por ende, en un aprendizaje que no está sujeto necesariamente a un interés por la recuperación y la preservación de la lengua o de la biodiversidad, sino a los hechos circunstanciales, la participación y la necesidad misma de recordar o aplicar estos conocimientos, es decir, consolidan un proceso de endoculturación.

La experiencia reflexiva del TEK de los matlatzincas se convierte en un referente para proponer estudios a profundidad e interdisciplinarios que complementen o profundicen dicho conocimiento y la biodiversidad, a través de estudios colaborativos entre las ciencias sociales y otras disciplinas para, por ejemplo, la determinación taxonómica de las plantas, el monitoreo y registro de la fauna silvestre, el desarrollo de planes de uso y manejo de los recursos forestales y la conservación de la biodiversidad. Asimismo, dicha experiencia reflexiva refuerza los primeros acercamientos a la relación que guardan las comunidades indígenas con su entorno natural y que afrontan procesos de cambio cultural influenciados por la modernización y que pueden derivar en pérdida o desuso del тек. Dichos acercamientos se vieron favorecidos por el uso de metodologías cualitativas basadas en principios éticos y de respeto hacia la comunidad indígena, permitiendo documentar el conocimiento, las prácticas y las creencias en torno a la biodiversidad de sus bosques, reflejados en sus testimonios.

\section{Conclusiones y sugerencias}

El acercamiento y la documentación de las formas locales de nombrar y entender el mundo contribuyen a la generación de conocimientos sobre biodiversidad que posibilitan la continuidad de

ojo y que su piel se utilizaba para llevar a cabo prácticas de "limpias" relacionadas con enfermedades provocadas por brujería. 
una cultura y la protección del patrimonio biocultural. En San Francisco Oxtotilpan los conocimientos, creencias y prácticas en relación con la biodiversidad son un bien intangible que pertenece a sus habitantes y que pueden contribuir de manera considerable al conocimiento de la biodiversidad a nivel local, regional, nacional e internacional. A través de la documentación del TEK se demuestra que aún existe una biodiversidad reconocida por los habitantes de la comunidad matlatzinca por satisfacer diversas necesidades de índole doméstica, económica, medicinal y ritual.

La incorporación del TEK a las estrategias de desarrollo sustentable con una dimensión cultural y ecológica podría reforzar y valorar los conocimientos tradicionales de los grupos indígenas para su inclusión en las políticas de conservación del patrimonio biocultural. Por tanto, la realización de estudios desde otras disciplinas y enfoques, como por ejemplo las determinaciones taxonómicas o sobre el uso y manejo de los recursos forestales maderables y no maderables, permitirán complementar los resultados del análisis del тек, que no solo permite la generación misma de conocimiento, sino también su aplicación en dichas políticas públicas, considerando las peculiaridades de las comunidades indígenas como los matlatzincas del altiplano central de México.

Agradecimientos: a la Universidad Autónoma del Estado de México, a través de la Secretaría de Investigación y Estudios Avanzados, por el financiamiento del proyecto de investigación "Conocer para conservar: la biodiversidad, un servicio ambiental en el Nevado de Toluca", clave 3561/2013cht, así como por la beca otorgada a Laura Millán Rojas para la elaboración de su tesis. 


\section{Referencias}

Berger, P. L. y Luckmann, T. (2011). La construcción social de la realidad. Buenos Aires: Amorrortu.

Berkes, F. (1993). Traditional Ecological Knowledge. En J. T. Inglis. (Eds.), Traditional Ecological Knowledge: Concepts and Cases (pp. 1-9). Ottawa: International Program on Traditional Ecological Knowledge-International Development Research Centre.

Berkes, F. (1999). Sacred Ecology. Traditional Ecological Knowledge and Resource Management. Philadelphia: Taylor \& Francis.

Borboa, A. (1999). Temascaltepec. Monografía Municipal. México: Instituto Mexiquense de Cultura.

Comisión Nacional de Áreas Naturales Protegidas (Conanp) (2014). Borrador del programa de manejo del APFF Nevado de Toluca para consulta pública. Recuperado el 25 de mayo del 2014, de http://www.conanp.gob. $\mathrm{mx} / \mathrm{anp} /$ consulta/BORRADOR\%20PM\%20NEVADO\%20DE\%20TOLUCA-311013.pdf.

Corsiglia, J. (2006). Traditional Wisdom as Practiced and Transmitted in Northwestern British Columbia, Canada.

En C. Menzies (Ed.), Traditional Ecological Knowledge and Natural Resource Management (pp. 221-235). Estados Unidos: Universidad de Nebraska.

Gaceta Municipal (2013). Plan de Desarrollo Municipal 2013-2015. México: Municipio de Temascaltepec.

Giménez, G. (1995). Modernización, cultura e identidad social. Espiral Estudios sobre Estado y Sociedad, 1(2), 35-56.

Hornborg, A. (2001). La ecología como semiótica. Esbozo de un paradigma contextualista para la ecología humana. En P. Descola y G. Pálsson (Coords.), Naturaleza y sociedad perspectivas antropológicas (pp. 60-79). México: SXXI.

Instituto Nacional de Estadística y Geografía (Inegi) (2010). Censo de población y vivienda 2010. Recuperado el 20 de octubre de 2013 de http://www.censo2010.org.mx/.

Lara, R. y Vides, R. (2014). Sabiduría y adaptación. El valor del conocimiento tradicional para la adaptación al cambio climático en América del Sur. Quito: Unión Internacional para la Conservación de la Naturaleza y de los Recursos Naturales.

LaRochelle, S. y Berkes, F. (2003). Traditional Ecological Knowledge and Practice for Edible Wild Plants: Biodiversity use by the Rarámuri in the Sierra Tarahumara, Mexico. International Journal of Sustainable Development \& World Ecology, 10, 361-375.

Latapí, A. (2009). Diversidad cultural: pueblos indígenas, situación y perspectivas. En G. Ceballos, R. List, G. Garduño, R. López Cano, M. J. Muñozcano, E. Collado y J. E. San Román (Comps.), La diversidad biológica del Estado de México. Estudio de Estado (pp. 63-72). México: Gobierno del Estado de México, Biblioteca Mexiquense del Bicentenario.

Magallanes, A. B., Limón, F. y Ayús, R. (2005). Nutrición de cuerpo y alma: practices y creencias alimentarias durante el embarazo en Tziscao, Chiapas. Revista Nueva Antropología, 131-148.

Manfredo, M. J., Teel, T. L., Gavin, M. C. y Fulton, D. (2014). Considerations in Representing Human Individuals in Social-Ecological Models. En M. J. Manfredo, J. J. Vazke, A. Rechkemmer y E. A. Duke (Eds.), Understanding Society and Natural Resources (pp. 137-158). Nueva York: Springer.

Mariaca, R., Cano, E. J., Morales, G. y Hernández, M. (2014). La milpa en la región serrana Chiapas-Tabasco de Huitiupán-Tacotalpa. En M. González y M. C. Brunel (Cords.), Montañas, pueblos y agua. Dimensiones y realidades de la Cuenca Grijalva (pp. 323-359). México: El Colegio de la Frontera Sur. 
Menzies, C. (2006). Ecological Knowledge, Subsistence, and Livelihood Practices. The Case of the Pine Mushroom Harvest in Northwestern British Columbia. En C. Menzies (Ed.), Traditional Ecological Knowledge and Natural Resource Management (pp. 87-104). Estados Unidos: Universidad de Nebraska.

Menzies, C. y Butler, C. (2006). Understanding Ecological Knowledge. En C. Menzies (Ed.), Traditional Ecological Knowledge and Natural Resource Management (pp. 1-17). Estados Unidos: Universidad de Nebraska.

Milton, K. (1996). Environmentalism and Cultural Theory. Exploring the role of anthropology environmental discourse. Nueva York: Routledge.

Moran, F. (2000). Human Adaptability. An Introduction to Ecological Anthropology. Colorado: Westview Press.

Naciones Unidas (1992). Convenio sobre la diversidad biológica. Recuperado el 5 de octubre del 2013, de https:// www.cbd.int/doc/legal/cbd-es.pdf.

Quezada, N. (1996). Los matlatzincas época prehispánica y época colonial hasta 1650. México: Universidad Autónoma de México.

Quiñonez, M., Ruan, F., Aguilar, I. E., Garza, F., Lebgue, T., Lavín, P. A. y Enríquez, I. D. (2014). Knowledge and Use of Edible Mushrooms in Two Municipalities of the Sierra Tarahumara, Chihuahua, Mexico. Journal of Ethnobiology and Ethnomedicine, 10(67), 1-13.

Ruiz, I. y Corbera, E. (2013). Community-Based Conservation and Traditional Ecological Knowledge: Implications for Social-Ecological Resilience. Ecology \& Society, 18(4).

Santos, D., Naranjo, E. J. y Rangel, J. L. (2012). Wildlife uses and hunting patterns in rural communities of the Yucatan Peninsula, Mexico. Journal of Ethnobiology and Ethnomedicine, 8(38), 1-17.

Saynes, A., Caballero, J., Meave, J. A. y Chiang, F. (2013). Cultural change and loss of ethnoecological knowledge among the Isthmus Zapotecs of Mexico. Journal of Ethnobiology and Ethnomedicine, 9(40), 1-10.

Toledo, V. (2005). La memoria tradicional: la importancia agroecológica de los saberes tradicionales. Leisa. Revista de Agroecología, 20(4), 16-19.

Toledo, V. y Barrera, A. (2009). A etnoecología: uma ciencia pós-normal que estuda as sabedorias tradicionais. Desenvolvimento e Meio Ambiente (20), 31-45.

Townsend, P. (2009). Environmental Anthropology. From Pigs to Policies. Estados Unidos: Waveland Press. 